\title{
Microwave Radiation Sterilization
}

National Cancer Institute

\section{Source}

National Cancer Institute. Microwave Radiation Sterilization. NCI Thesaurus. Code

C101694.

A sterilization process that uses microwave radiation to kill microorganisms. This process

is restricted to materials that contain water and is typically used for food. 